\title{
Role of Vacuum assisted Negative pressure wound therapy in management of Enterocutaneous fistulae arising within the open abdomen: three case studies.
}

\author{
Gopalakrishnan $\mathrm{G}^{1}$, Debasis Naik ${ }^{1}$, Megha Tandon ${ }^{2}$, Deepak Rajput ${ }^{2}$ \\ ${ }^{I}$ (Post-Graduate, Department of General Surgery, V.M.M.C. and Safdarjung Hospital, New Delhi, India) \\ ${ }_{2}^{2}$ (Senior Resident, Department of General Surgery, V.M.M.C. and Safdarjung Hospital, New Delhi, India)
}

\begin{abstract}
An Enterocutaneous fistulae (ECF) also known Gastro-intestinal fistulae, communicates between stomach, small or large bowel and skin, allowing gastrointestinal contents to flow on to the skin. The majority of ECF (75\% - 90\%) are the result of previous surgeries but it is estimated that 10\% - 25\% of the patients may develop an ECF without having had a surgical procedure. Negative pressure wound therapy (NPWT) is used extensively in the acute and chronic wound care arena of health care. It has become a standard of care for many types of wound from non-healing diabetic foot ulcer and burns to traumatic and surgical wounds. Recently attention has been paid using this system for the treatment of catastrophic abdominal wounds and open abdomen with ECF. We present 3 cases of ECF arising within open abdomen managed successfully by Vacuum assisted Negative pressure wound therapy.
\end{abstract}

Keywords: Enterocutaneous fistulae, Negative pressure wound therapy, Open abdomen, Vacuum assisted closure.

\section{INTRODUCTION}

Enterocutaneous fistulae (ECF) represent an abnormal communication between abdominal hollow viscera and abdominal wall, and present a difficult problem for the patients and clinicians[1]. ECF may lead to a multitude of untoward consequences including nutrition deficiencies, volume depletion, septic complication and long term wound management problems[2]. Negative pressure wound therapy (NPWT) have the following benefits in management of ECF: improved spontaneous closure rate, a good patient safety profile, economic viability of this therapy, relative simplicity of this treatment and decreased length of hospital stay[3]. We report 3 cases of ECF arising within open abdomen managed successfully by Vacuum assisted Negative pressure wound therapy.

\section{Method}

A polyurethane bag was taken and its tube end was cut keeping the size of the bag enough to cover the wound with a minimum of $5 \mathrm{~cm}$ margin on all sides. Multiple $1 \times 1 \mathrm{~cm}$ fenestrations were made at the centre of the inferior film of the bag. Now the edge and surrounding skin were cleaned and dried. Super glue adhesive was applied to the edge of the wound and the surrounding skin and the bag was pasted over the anterior abdominal wall keeping the fenestrations over the wound and maintaining appropriate traction so as to make the wound contract. The distal portion of a 18 Fr Ryle's tube was placed into the pouch of the polyurethane bag after increasing the size of its fenestrations. The opening of the bag was then closed with glue and then an adhesive drape sheet was used to cover the bag with the anterior abdominal wall (Fig 1). The proximal end of the Ryle's tube was connected to a vacuum system maintained at -100 to $-150 \mathrm{~mm}$ of $\mathrm{Hg}$ intermittently (5min suction followed by 2 min off suction). The dressing was changed on every third day and as and when required.

\subsection{Case 1}

\section{Case Reports}

A 27 year old male, presented to our casualty in septicemic shock with faeculent discharge from an open midline abdomen wound along with a non-functioning loop ileostomy on the right side. He had history of Exploratory laparotomy and loop ileostomy with abdominal lavage for ileal perforation peritonitis 10 days back. Due to his poor APACHE II score and general condition he was planned for conservative management. He was treated as per the standard protocol of ECF management along with Vacuum assisted negative pressure dressing over the open midline wound. Patient gradually recovered from sepsis, the output from the midline wound gradually decreased from 1.5 litres on day 1 to $5-10 \mathrm{ml}$ at the end of 3 weeks. His ileostomy also started functioning around the $3^{\text {rd }}$ day. Patient was discharged from the hospital at the end of $4^{\text {th }}$ week with a healed midline scar and a functioning ileostomy (Fig 2 shows the wound of the patient on $22^{\text {nd }}$ day) with advice to 
follow up with CECT abdomen report. CECT abdomen revealed no abnormal communication between the bowel and the anterior abdominal wall except ileostomy.

\subsection{Case 2}

A 25 year old female, presented to us with faecal matter discharge per vaginum and foul smelling discharge from lower midline wound. She had underwent LSCS 7 days back. Patient was taken up for exploratory laparotomy, intraoperatively terminal ileum was found partially transected with gaping of uterine wound. Primary repair of uterine wound with loop ileostomy at the site of transection with Bagota Bag procedure was done. On the fifth post-op day, she developed faecal matter discharge from the midline wound and also per vaginum. On examination, there was a $0.5 \times 0.5 \mathrm{~cm}$ perforation in the bowel present in the lower part of the midline wound with faecal matter coming out of it. She was also treated as per the standard protocol of ECF management along with Vacuum assisted Negative pressure dressing and Vaginal douche. Gradually, faecal matter discharge per vaginum decreased from $200 \mathrm{ml}$ on day 1 to nil at the time of discharge (6 weeks). The discharge from midline wound reduced from 1 litre on day one to around $500 \mathrm{ml}$ at the time of discharge, also the fistula became a controlled one (Fig 3 shows the wound of the patient on $25^{\text {th }}$ day). Output from the loop ileostomy remained unchanged $100-200 \mathrm{ml} /$ day throughout the period of hospital stay.

\subsection{Case 3}

A 37 year old female underwent double barrel ileostomy closure. On the $5^{\text {th }}$ postoperative day, patient developed burst abdomen followed by faeculent discharge from the midline wound. The same standard protocol of ECF management was followed along with Vacuum assisted negative pressure dressing. The output from the midline wound decreased from $700 \mathrm{ml}$ on day 1 to $100 \mathrm{ml}$ after 2 weeks (Fig 4 shows the wound of the patient on $8^{\text {th }}$ day). Patient was discharged home with a controlled fistula in the midline with an ileostomy bag.

\section{Discussion}

A fistula is an abnormal opening between two or more organs or structures. The aetiology of fistula formation varies widely according to the organs involved, precipitating factors, patients risk factors, and surgical technique or procedure[4].

Most (75 to 90\%) ECFs are iatrogenic or, to be more specific, postoperative or postprocedural[4,5,6,7,]. Approximately half of these are thought to be caused by anastomotic leak or dehiscence and about half by inadvertent enterotomy[4]. The other 10 to 25\% of ECFs are spontaneous. These include fistulas from inflammatory bowel disease, neoplasia, vascular insufficiency such as radiation enteritis or mesenteric ischemia, diverticulitis, appendicitis, pancreatitis, tuberculosis, or other intra-abdominal infections, abscesses, or inflammatory processes such as malakoplakia[8]. Penetrating traumatic fistulas are included in the spontaneous category[7].

The complexity of an enterocutaneous fistula depends upon the volume and the nature of the output. Lowvolume output is $<200 \mathrm{ml} / 24$ hours, moderate output is 200 to $500 \mathrm{ml} / 24$ hours and high output is $>500 \mathrm{ml} / 24$ hours[4].

Schein and Decker cited 37\% mortality rate in post-operative high output ECF. The majority of these deaths are attributed to electrolyte imbalance, malnutrition and sepsis[9].

Early CT is the study of choice in patients with signs of sepsis, as it may delineate areas of undrained abscess. Repeating the $\mathrm{CT}$ as needed after percutaneous drainage to ensure complete drainage is recommended. These scans when performed with oral contrast material may also demonstrate the fistula tract and provide anatomic information about the orientation and length of the tract and its "feeding bowel"[10]. The "gold standard" for examining a fistula is a fistulogram performed with water-soluble contrast material. This should be performed later in the management of the fistula, after stabilisation and treatment of sepsis. A fistulogram can show the configuration of the tract, the source of fistula, and the abscess cavity that communicates with the fistula[11]. Ultrasonography can demonstrate involved bowel, abscess cavities, and areas of possible stenosis but does not adequately evaluate the fistula tract[12]. Endoscopy can be used in the accumulation of data regarding the characteristics of a fistula and surrounding bowel[13].

Spontaneous closure of a colonic fistula can take 30-40 days; an ileal fistula 40-60 days. Ninety percent of enteric fistulas that do close will do so within 50 days[14,15].

The strategies for management of ECF include nutritional support, correction of electrolyte imbalances, recognition and treatment of sepsis, localisation and delineation of the anatomy of the fistula and a correctly timed operative procedure. Newer therapies including wound vacuum assisted closure (VAC), fibrin glue and the use of somatostatin analogues have been used to promote the closure of ECF[16].

In the late 1990s the VAC system was thought to promote or at least potentiate fistulas and was not used for this indication; however, there has been a resurgence of interest and use in this device for fistulas. The VAC system can be tailored in many ways to divert fistula effluent. As described by Goverman et al, the VAC also treats the wound bed around the fistula with negative-pressure dressing, resulting in increased granulation 
tissue and wound contracture[17]. Majority of the authors, usually apply a constant VAC mode, usually 125 $\mathrm{mmHg}$ at an intermittent pressure cycle of 5 min suction followed by 2 min off suction[18].

The VAC system is thought to work by several different mechanisms. Active removal of excess interstitial fluids from tissues may decompress small blood vessels allowing incremental increases of blood flow and therefore improve supply of oxygen and nutrients for tissue repair. the increased blood flow speeds up granulation tissue formation by $63 \%$ over non-VAC treated wounds. Mechanical stress may also play a part by switching on a mechanism which increases cellular proliferation and angiogenesis similar to ilizarov technique. The VAC also leads to reduced bacterial colonisation by anaerobic organisms through increasing tissue oxygen concentrations. Neutrophils use the increased oxygen to kill bacteria. Bacterial colonisation was decreased by 1000 -fold compared with non-negative pressure exposed wounds after four days of treatment. The VAC system could prove to be an extremely valuable tool to study changes in the wound microenviroment by analysing timed aliquots of the fluid removed[19].

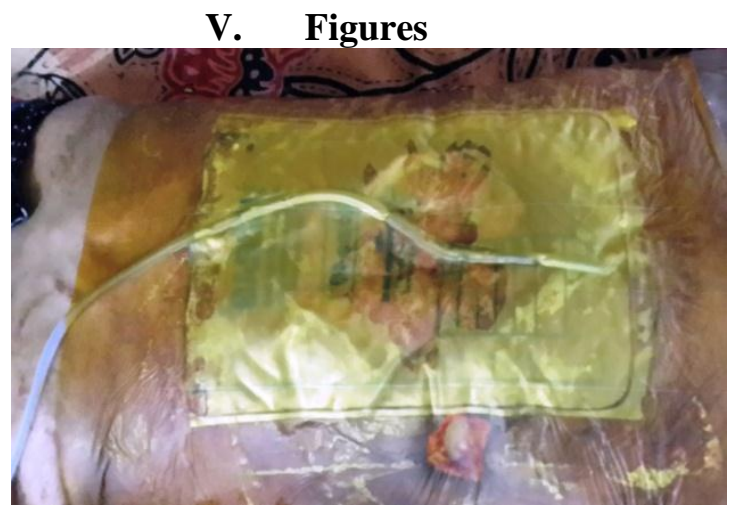

Fig 1 : Vacuum assisted negative pressure dressing system using polyurethane bag, Ryle's tube and sterile adhesive drape sheet.

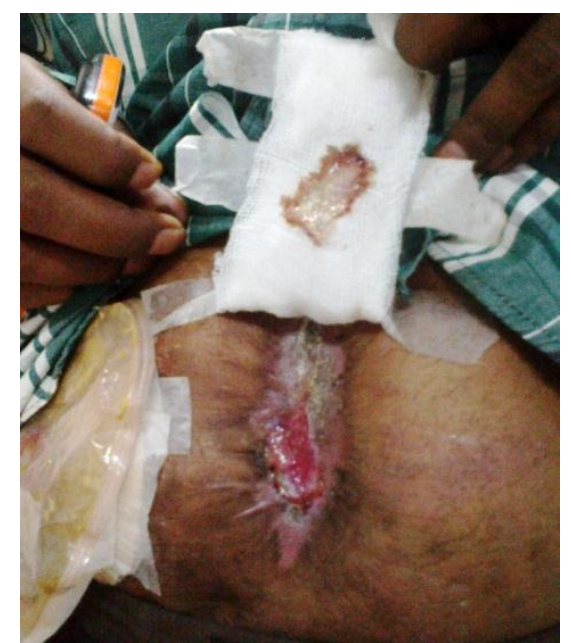

Fig 2 : wound of the patient in case 1 on $22^{\text {nd }}$ day of NPWT showing healing mid line wound and functioning stoma on the right.

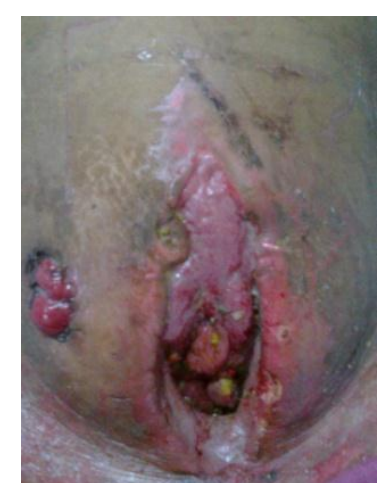

Fig 3 : wound of the patient in case 2 on $25^{\text {th }}$ day of NPWT showing healing mid line wound with granulation tissue, a controlled fistula at the lower part of the wound and ileostomy on the right. 


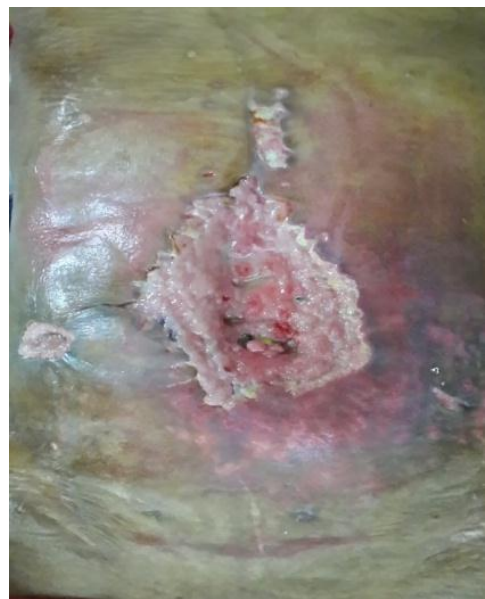

Fig 4 : wound of the patient in case 3 on $8^{\text {th }}$ day of NPWT showing well formed granulation tissue with a fistula at its lower part.

\section{Conclusion}

In conclusion, Vacuum assisted negative pressure therapy is an effective adjunct treatment in the management of ECF. It decreases the rate of infection, promotes spontaneous closure, improves skin integrity around the fistula site and lowers the requirement of dressing changes, especially in high output fistulae.

\section{REFERENCES}

[1]. Satwicki SP, Braslow BM. Gastrointestinal fistulae. OPUS 12 Scientist 2008; 2(1): 13-16.

[2]. Denham DW, Fabri PJ. Enterocutaneous fistula. In: Cameron JL, Ed. Current Surgical Therapy, $7^{\text {th }}$ ed. St Louis: Mosby. 2001.

[3]. J. Cipolla et al. Negative pressure wound therapy: Unusual and Innovative applications. OPUS 12 Scientist. 2008; 2 (3); 15-29.

[4]. Berry SM \& Fischer JE. Classification and pathophysiology of enterocutaneous fistulas. Surg Clin North Am 1996; 76: 1009-1018.

[5]. Cameron J. Current Surgical Therapy. $7^{\text {th }}$ ed. St. Louis, MO: Mosby; 2001. Pp. 156-161.

[6]. Haffejee A A. Surgical management of high output enterocutaneous fistulae: a 24-year experience. Curr Opin Clin Nutr Metab Care. 2004; 7: 309-316.

[7]. Makhdoom Z A, Komar M J, Still C D. Nutrition and enterocutaneous fistulas. J Clin Gastroenterol. 2000; 31: 195-204.

[8]. Sinha S K, Sethy P K, Kaman L, et al. Multiple spontaneous enterocutaneous fistulae in malakoplakia. Indian J Gastroenterol. 2003; 22: $234-235$

[9]. Schein M, Decker G A. Postoperative external alimentary tract fistulas. In Bryant R (ed.), Acute and Chronic Wounds. St. Louis: Mosby. 1991: 74.

[10]. D’Harcour J B, Boverie J H, Dondelinger R F. Percutaneous management of enterocutaneous fistulas. AJR Am J Roentgenol. 1996; 167: 33-38.

[11]. Evenson A R, Fischer J E. Current management of enterocutaneous fistula. J Gastrointest Surg. 2006; 10: $455-464$.

[12]. Marconi G, Parente F, Porro G B. Hydrogen peroxide enhanced ultrasound - fistulography in the assessment of enterocutaneous fistulas complicating Crohn's disease. Gut. 1999; 45: 874-878.

[13]. Regueiro M. The role of endoscopy in the evaluation of fistulizing Crohn's disease. Gastrointest Endosc Clin N Am. 2002; 19: 230236.

[14]. Allardyce D B. Management of small fistulas. In Bryant R (ed.), Acute and Chronic wounds. St. Louis: Mosby. $1983: 321$

[15]. Berry S M, Fischer J E. Classification and pathophysiology of enterocutaneous fistulas. In Bryant R (ed.), Acute and Chronic wounds. St. Louis: Mosby. 1996: 321.

[16]. Deepa Taggarshe, Daniel Bakston, Michael Jacobs, Alasdair McKendrick, Vijay Mittal. Management of enterocutaneous fistulae: A 10 years experience. World J Gastrointest Surg. 2010; 2 (7): 242-246.

[17]. Goverman J, Yelon J A, Platz J J, Singson R C, Turcinovic M. The "fistula VAC" a technique for management of enterocutaneous fistulae arising within the open abdomen: report of 5 cases. J Trauma. 2006; 60: 428-431.

[18]. Venture M L, Attinger C E, Mesbahi A N, et al. Mechanisms and clinical application of vacuum-assisted closure (VAC) device: a review. Am J Clin Dermatol 2005; 6: 185-94.

[19]. Morykwas M J, Argenta L C. Nonsurgical modalities to enhance healing and care of soft tissue wounds. J South Orthop Assoc 1997; 6: 284-6. 